\title{
LV-RV Shape Modeling Based on a Blended Parameterized Model
}

\author{
Kyoungju Park ${ }^{1}$, Dimitris N. Metaxas ${ }^{1,2}$, and Leon Axel ${ }^{3}$ \\ 1 Department of Computer and Information Science \\ University of Pennsylvania, Philadelphia, PA 19104, USA \\ kypark@graphics.cis. upenn.edu \\ 2 CBIM Center, Computer Science Department and Bioengineering Department \\ Rutgers University, New Brunswick, NJ 08854, USA \\ dnm@cs.rutgers.edu \\ 3 Department of Radiology \\ New York University School of Medicine, New York, NY10016, USA
}

\begin{abstract}
Making a generic heart deformable model to be able to analyze normal and pathological hearts is important. Such a generic model gives more stability and accuracy for segmentation, analysis and classsification. Due to the conflicting demands of shape generality and shape compactness, such a generic heart model is difficult to define. In order to be useful, a generic heart model should be defined with a few number of parameters. In all the previous work on the modeling of the LV-RV shape the deformable model is built from the given datasets. Therefore such methods have limitations that the quality of shape estimation are dependent on the quality of the datasets. In this paper, we introduce a blended deformable model approach with parameter functions which is generic enough to deal with the different heart shapes. Using a method we are able to model the 3D shape of the heart which include the left ventricle(LV) and the right ventricle(RV). We also include the inflow and outflow tract of the RV basal area, so that the full LV-RV shape can be estimated.
\end{abstract}

\section{Introduction}

Making a generic heart deformable model to be able to analyze normal and pathological hearts is important. We can estimate heart shape automatically with a generic model so that we do not have a problem with a tedious mesh generation process depending on segmented datasets. Also we can compare the different heart shapes based on a generic model. Such a generic model gives more stability and accuracy for segmentation, analysis and classification.

Due to the conflicting demands of shape generality and shape compactness, a generic heart model is difficult to define. In shape estimation and motion analysis, the various shapes, morphological changes and deformation over time should be covered.

On the other hand, a compact description is important. Features of the heart shape and motion should be represented quantitatively in terms of relatively few descriptors. Thus it is very easy to compare different heart shapes. 
In this paper, we introduce a blended deformable model approach with parameter functions which are generic enough to deal with the different heart shapes. Using this method we are able to model the 3D shape of the heart which include the left ventricle(LV) and the right ventricle(RV).

This blending operation on deformable models allows the combination of two different shape primitives in one single model. The parameter functions describe the object with a few parameters while allowing variations by those parameter functions. In the past, the parameter functions are used to model the LV 910 . Now we extend to the LV-RV model with a blended model. The blended shape is employed more to cover not only the LV but also the more complicated right ventricle(RV) and the up basal area.

The heart boundary datasets are extracted from our MRI-SPAMM imaging technique [1] which has been shown to be the best non-invasive in-vivo motion study tool. In shape estimation, we use the physics-based framework of Metaxas and Terzopoulos [7. The heart shape model deforms due to forces exerted from the datasets.

Our method of a blended deformable model with parameter functions automatically generates the FEM meshes and is generic enough to deal with the normal and pathological hearts. Thus we can compare the different hearts based on the generic heart model.

In section 2 and 3, we will explain how to define a generic heart shape model and how to estimate the shape using physics-based framework. Then section 4 shows the reconstructed heart models of normal subjects and patient studies.

\subsection{Related Work}

Many approaches have been developed for making a heart model. Such models usually have a number of parameters to control the shape and pose of all or part of the model.

Park et al.109 used the parameter functions with the generalization of an ellipsoid primitive for LV motion analysis based on MRI-SPAMM. Similarly Young [1] build a heart model using the prolate spheroidal coordinates. This model is used to the direct motion traking from tagged images. Declerck et al. [3] use the 4D planispheric transformation for tracking and motion analysis of LV. Papademetris et al. 8 use the active elastic model for regional cardiac deformation from MR and the 3D Echocardiography. All these approaches, however, are applied only to the LV rather than the whole LV-RV.

Only a few approaches are made to build the LV-RV model. Haber et al. [4] reconstructed the LV-RV motion. It is the only model so far that constructed the RV motion. However, this heart model depends on the quality of the input data. For every dataset, the different meshes should be generated and that makes it difficult to compare the different hearts. It is therefore difficult to be used for analyzing, classifying and comparing large datasets. 


\section{Shape Modeling}

We introduce the use of a blended model with parameter functions. Blended shapes and boolean operations have been used in geometric model and recently exploited in free-form solids[6] and deformable models[2].

\subsection{Blended Deformable Shape Model}

The blended shape is composed of several primitive parts by use of the blending function. The component primitives we pick are the deformable primitives with a small number of parameters. Examples are generalized cylinders, geons, superquadrics, hyperquadrics etc.

With the structured shape representation scheme, the single blended shape model can be thought of as the combination of two deformable primitive parts. Portions of component primitives are cut out, and the selected portions are joined together.

Our blended shape model combines portions from component primitives $s 1$ and $s 2$. The resulting created model, $s$, has a global single coordinate system $(u, v)$ (latitude $u$, longitude $v$ ). Thus we can define $s$ as the new class of deformable primitives.

For example, something shaped like a half-moon can be built from two ellipsoid like primitives. Firstly, we define underlying deformable primitives $s_{1}(u, v)$ and $s_{2}(u, v)$ as follows:

$$
s_{1}(u, v)=\left(\begin{array}{c}
r_{11} \cos u \cos v \\
r_{12} \cos u \sin v \\
r_{13} \sin u
\end{array}\right), s_{2}(u, v)=\left(\begin{array}{c}
r_{21} \cos u \cos v \\
r_{22} \cos u \sin v \\
r_{23} \sin u
\end{array}\right)
$$

where $-\pi / 2 \leq u \leq \pi / 5,-\pi \leq v<\pi$. The cut-and-paste operations on $s_{1}(u, v)$ and $s_{2}(u, v)$ are placed over the $(u, v)$ coordinates level. This allows blended shape $s$ to have global parameterization without reparameterizing. Our created blended shape $s$ in Fig. 1 is defined as follows:

$$
s(u, v)= \begin{cases}s 1(u, v) & \text { if } 0<v<\pi \\ s 2(u,-v) & \text { if }-\pi<v<0 \\ (s 1(u, v)+s 2(u,-v)) / 2 & \text { if } v=0, v=-\pi\end{cases}
$$

where $-\pi / 2 \leq u \leq \pi / 5,-\pi \leq v<\pi$.

In addition to blending we allow further local variations on the underlying shape by replacing the parameters of deformable primitives with functions. This allows the flexibility of capturing specific variations while keeping the guidance of the generic model.

\section{$2.2 \quad$ Heart Model Geometry}

We apply the method of blending with parameter functions to build a LV-RV generic model. The heart is actually two separate pumps: a right heart and a 


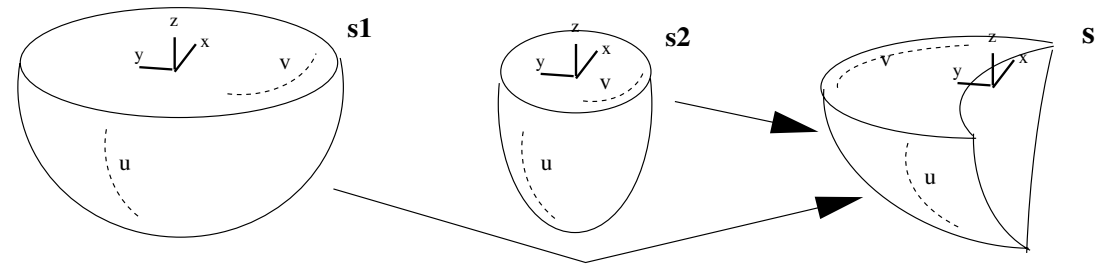

Fig. 1. Blended shape $s$ from shape $s 1$ and $s 2$

left heart. Each of these hearts is a two-chamber pump composed of an atrium and a ventricle.

To build the whole ventricle including the LV and RV with the material coordinates $\mathbf{u}=(u, v, w)$, we define a heart shape model $x(\mathbf{u})=\mathbf{c}+\mathbf{R} s(\mathbf{u})$ where $\mathbf{c}$ and $\mathbf{R}$ are the global translation and rotation of the model, and $s(\mathbf{u})$ is a blended model where $\mathbf{u}$ is taken from $(u, v, w)$ coordinates representing longitude, latitude and number of primitives respectively. Therefore $\mathbf{s}(\mathbf{u})$, the blended model, presents the position of the points on the model relative to the model frame. The underlying deformable primitive $e(\mathbf{u})$ is a generalization of an ellipsoid primitive such that piecewise functions of $\mathbf{u}$ take the place of parameters, as follows:

$$
e(\mathbf{u})=\left(\begin{array}{c}
x \\
y \\
z
\end{array}\right)=s c\left(\begin{array}{c}
r_{1}(\mathbf{u}) \cos u \cos v \\
r_{2}(\mathbf{u}) \cos u \sin v \\
r_{3}(\mathbf{u}) \sin u
\end{array}\right)
$$

where $-\pi / 2 \leq u \leq \pi / 5,-\pi \leq v<\pi, w>0, r_{1}(\mathbf{u}), r_{2}(\mathbf{u}), r_{3}(\mathbf{u}) \geq 0 ; s c$ is the scaling parameter for the model and $r_{1}(\mathbf{u}), r_{2}(\mathbf{u})$ and $r_{3}(\mathbf{u})$ are the $x, y, z$ axial deformation parameters respectively.

Our heart shape model is composed of three layers $(w=1,2,3)$; LV endocardium, epicardium and RV endocardium respectively. The origin of model frame is the center of LV. The principal axis ( $z$-axis) of heart model is the line that connects the center of the LV apex and the center of the LV base and the $y$-axis is the line that connects the center of the the LV and the center of the RV.

The LV endocardium and epicardium are defined in (3). Each $\left(r_{1}, r_{2}, r_{3}\right)$ has two values : positive direction parameter $\left(r_{1+}, r_{2+}, r_{3+}\right)$ and negative direction parameter $\left(r_{1-}, r_{2-}, r_{3-}\right)$ along $x, y, z$ axes. The RV endocardium, unlike LV endocardium and epicardium, is a blended shape. As in (2), we create a blended model from two component primitives defined in (3). When shaping the RV endocardium, we cut-and-paste the selected portions of the above given deformable primitive $e$, as follows:

$$
s_{1}(u, v, 3)= \begin{cases}e\left(u, r_{s}(\mathbf{u}) v+r_{t}(\mathbf{u}), 3\right) & \text { if } 0 \leq v<\pi \\ e\left(u,-r_{s}(\mathbf{u}) v+r_{t}(\mathbf{u}), 4\right) & \text { if }-\pi \leq v<0\end{cases}
$$

where $0<r_{s}(\mathbf{u})<1,0<r_{t}(\mathbf{u})<\pi ; r_{s}(\mathbf{u})$ is proportion(the arc length ratio of the septum) parameter function on $x y$-plane and $r_{t}(\mathbf{u})$ is the angle between the end of the septum and the $x$-axis on the $x y$-plane. 

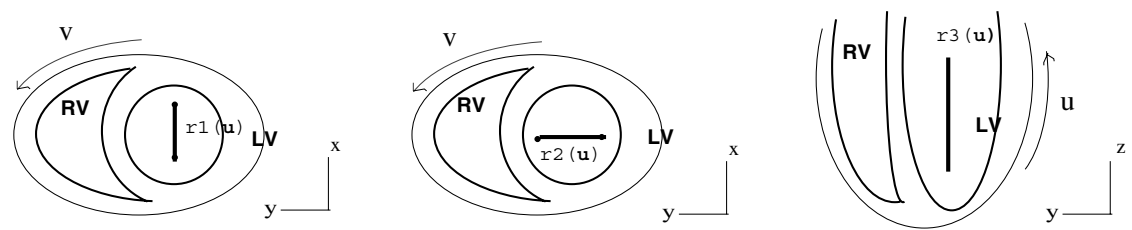

(a) Axial deformation parameters, $\mathrm{r} 1(\mathbf{u}), \mathrm{r} 2(\mathbf{u}), \mathrm{r} 3(\mathbf{u})$ along $\mathrm{x}, \mathrm{y}, \mathrm{z}-$ axis respectively
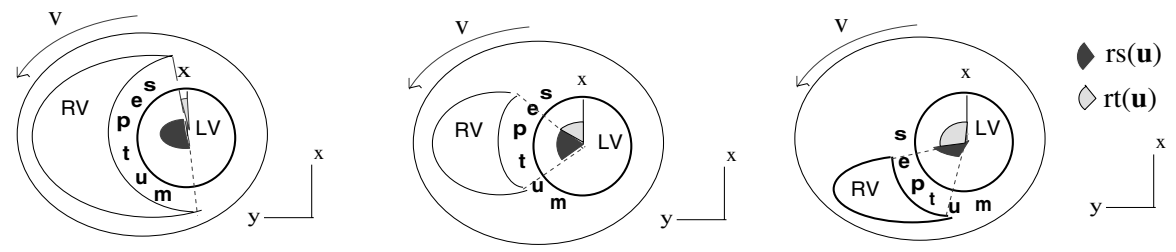

(h) Various sentum asnect ratio re(u) and sentum rotation rt(u) narameters
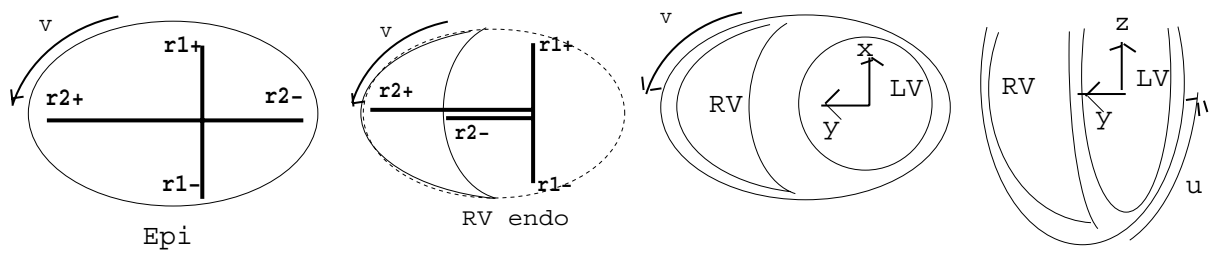

Fig. 2. Shape Model Parameters; Axial Deformation, Blending

Thus, the resulting volumetric blended heart shape model $s$ is:

$$
s(\mathbf{u})=\left\{\begin{array}{l}
s_{1}(\mathbf{u}) \text { if } w=3 \\
e(\mathbf{u}) \text { if } w=1,2
\end{array}\right.
$$

where $w=1,2,3$ represent LV endocardium, epicardium and blended RV endocardium. Therefore the blended model parameter vector $\mathbf{q}_{s}$ is defined as

$$
\mathbf{q}_{s}=\left(r_{1}(\mathbf{u}), r_{2}(\mathbf{u}), r_{3}(\mathbf{u}), r_{s}(\mathbf{u}), r_{t}(\mathbf{u})\right)
$$

The deformable shape model parameters are $\mathbf{q}=\left(\mathbf{q}_{c}^{T}, \mathbf{q}_{\theta}^{T}, \mathbf{q}_{s}^{T}\right)^{T}$, where $\mathbf{q}_{c}=c$ is the global translation, and $\mathbf{q}_{\theta}$ is the quaternion that states global rotation matrix $\mathbf{R}$.

\section{$3 \quad$ Fitting}

With our heart model geometry, we fit the model to the boundary datasets from our MRI-SPAMM [1 imaging technique. The heart imaging is taken from 13 short-axis views and 9 long-axis views for 7 time frames during systole. In $2 \mathrm{D}$ images we segment the LV-RV boundary using the active contours, snakes [5]. Based on those boundary datapoints we build the 3D heart model. 

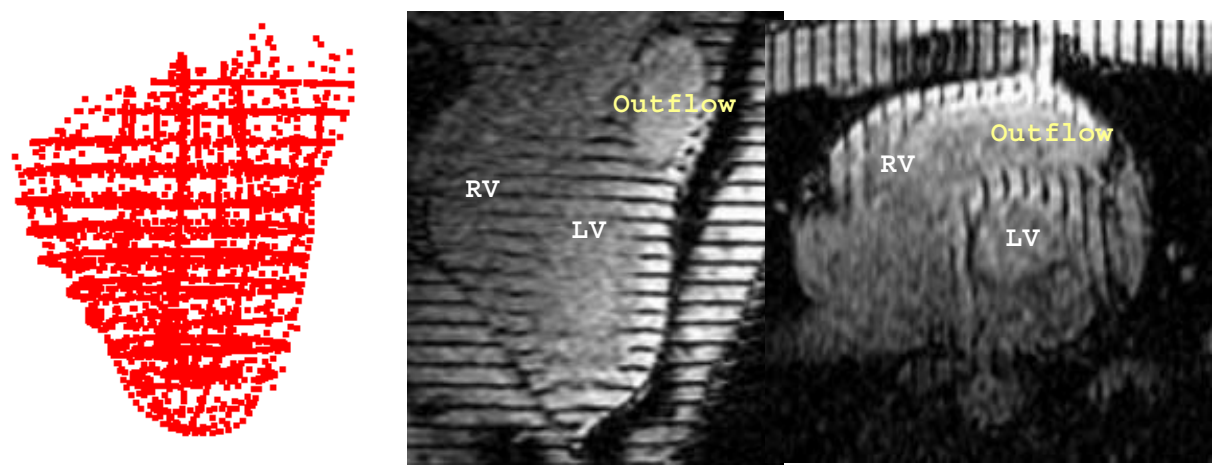

Fig. 3. Heart boundary data from MRI-SPAMM images

\subsection{Heart Model Dynamics}

The shape reconstruction process is done using the physics-based framework of Metaxas and Terzopoulos [7] with the geometrically defined model. Using this framework, a model deforms due to forces from datasets so as to minimize the applied forces. We use data forces to esimate the model parameters.

Estimation of the parameters is based on the simplified version of the Lagrangian dynamics equation of motion given by:

$$
\dot{\mathbf{q}}=\mathbf{f}_{\mathbf{q}}
$$

where $\mathbf{q}$ is the vector of shape parameters of the model and $\mathbf{f}_{\mathbf{q}}$ is the parameter forces which are determined from the $3 \mathrm{D}$ forces, $\mathbf{f}$ from datasets:

$$
\mathbf{f}_{\mathbf{q}}=\int \mathbf{L}^{\mathbf{T}} \mathbf{f}
$$

The Jacobian matrix $\mathbf{L}$ converts the $3 \mathrm{D}$ data forces $\mathbf{f}$ into forces which directly affect the parameters of the model[7]. The data forces, from each boundary point to the corresponding model wall, are computed by distributing force weights to the closest triangular element on the corresponding wall[10].

\subsection{Shape Estimation Process}

To fit the model to the data, the model is translated and rotated to the center of the mass of boundary datasets. Then we approximate the global shape and estimate the further local shape variations. With this hierarchical estimation, we can capture LV-RV shape accurately with enough degrees of freedom.

Our heart model with material coordinates $\mathbf{u}=(u, v, w)$ estimates the shape as follows: 
1. initialize a heart model with material coordinates

2. compute global translation and rotation : translate model to the center of the boundary datapoints rotate model so that $z$-axis set to the principal axis of the heart and $y$-axis points toward the RV

3. estimate scaling parameter: $s c$

4. estimate axial parameters for each wall $w=1,2,3$ and on positive and negative directions along $x y z$-axes:

$r_{1+}, r_{1-}, r_{2+}, r_{2-}, r_{3+}, r_{3-}$ for LV endocardium, RV endocardium and epicardium

5. compute blending parameters along $u$ for RV endocardium wrt model frame: the septum arc length ratio $r_{s}$ and septum rotation parameter $r_{t}$

6. estimate the piecewise axial parameters along $u, w$ :

$r_{1+}, r_{1-}, r_{2+}, r_{2-}, r_{3+}, r_{3-}$

7. estimate the local variations on all the material coordinates $(u, v, w)$ :

\section{Result}

Our blended model approach with parameter functions describe the heart shape quantitatively with translation, rotation, scaling, axial deformation and blending parameters. These parameters allow enough DOFs to deal with normal and pathological hearts and therefore are good to compare the different heart shapes. We applied the methods to the two normals and one abnormal datasets and we can see the differences in shapes and parameters.

Table 1. Axial Deformation Parameters r1, r2, r3 (mm)

\begin{tabular}{|l||r|r|r|r|r|r|r|r|r|}
\hline \multicolumn{1}{|c||}{} & \multicolumn{2}{c|}{ r1+ } & \multicolumn{2}{c|}{ r1- } & \multicolumn{2}{c|}{ r2+ } & \multicolumn{2}{c|}{ r2- } & \multirow{2}{*}{ r3 } \\
\cline { 2 - 9 } & apex & base & apex & base & apex & base & apex & base & \\
\hline \hline LV endo & 9.23 & 11.70 & 10.07 & 12.32 & 7.87 & 10.69 & 9,83 & 11.12 & 64.07 \\
\hline RV endo & 11.88 & 14.53 & 17.37 & 24.22 & 27.70 & 33.98 & 14.25 & 16.18 & 67.67 \\
\hline Epicardium & 13.33 & 15.82 & 17.11 & 23.61 & 29.27 & 35.64 & 14.86 & 16.43 & 70.70 \\
\hline
\end{tabular}

Table 1 is the typical parameters extracted from normal hearts. Figure 4 shows a reconstructed heart model at end-diastole phase of a normal subject. Figure 5 is RV hypertrophy patient model.

\section{Conclusion}

We have shown how to build a generic heart model including the RV and its outflow tract. Furthermore, the analysis of the shape parameters can be applied 

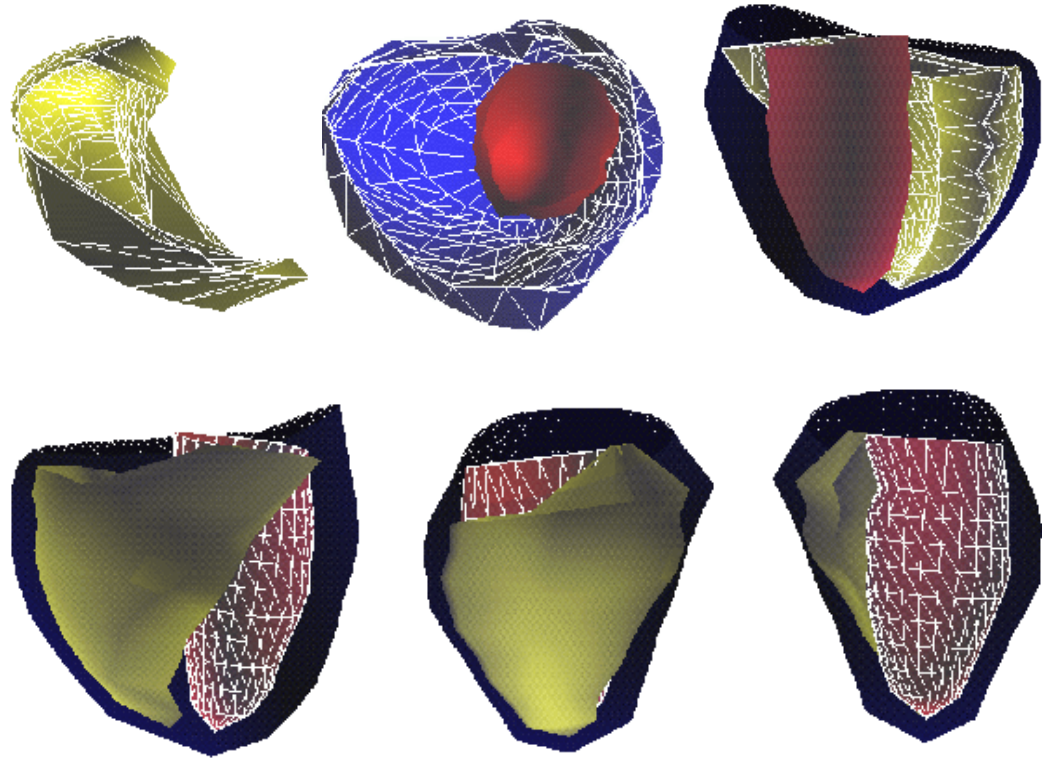

Fig. 4. LV-RV Heart Model at end-diastole
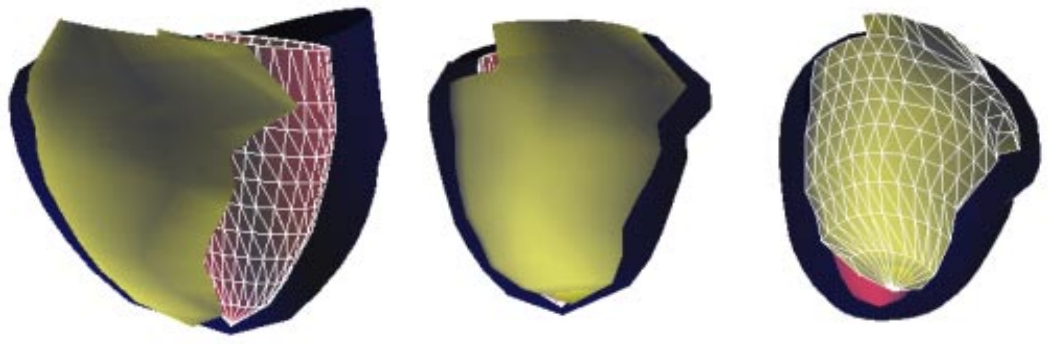

Fig. 5. RV Hypertrophy Model at end-diastole

to the morphological study of the shape. Future study will be the motion reconstruction and analysis of the heart from the MRI-SPAMM data. After a large number of experiments we will be able to define the parameters associated with normal motion and parameters associated with abnormal motion.

\section{References}

1. L. Axel and L. Dougherty. Heart wall motion: Improved method of spatial modulation of magnetization for mr imaging, 1989.

2. D. DeCarlo and D. Metaxas. Blended deformable models. In Proceedings of the Conference on Computer Vision and Pattern Recognition, pages 566-572, Los Alamitos, CA, USA, June 1994. IEEE Computer Society Press. 
3. Jerome Declerck, Nicholas Ayache, and Elliot R. McVeigh. Use of a 4D planispheric transformation for the tracking and the analysis of LV motion with tagged MR images. Technical Report RR-3535, Inria, Institut National de Recherche en Informatique et en Automatique.

4. Edith Haber, Dimitris N. Metaxas, and Leon Axel. Motion analysis of the right ventricle from MRI images. Lecture Notes in Computer Science, 1496:177-??, 1998.

5. Michael Kass, Andrew Witkin, and Demetri Terzopoulos. Snakes: Active Contour Models . Academic Publishers, 1987.

6. Daniel Kristjansson, Henning Biermann, and Denis Zorin. Approximate boolean operations on free-form solids. In Proceedings of ACM SIGGRAPH 2001, Computer Graphics Proceedings, Annual Conference Series, pages 185-194. ACM Press / ACM SIGGRAPH, August 2001. ISBN 1-58113-292-1.

7. D. Metaxas and D. Terzopoulos. Shape and nonrigid motion estimation through physics-based synthesis, 1993.

8. X. Papademetris, A. J. Sinusas, D. P. Dione, and J. S. Duncan. Estimation of 3D left ventricular deformation from echocardiography. Medical Image Analysis, 2001.

9. J. Park, D. Metaxas, and L. Axel. Analysis of left ventricular wall motion based on volumetric deformable models and MRI-SPAMM. Medical Image Analysis, 1(1):53-71, 1996.

10. J. Park, D. Metaxas, A. Young, and L. Axel. Deformable models with parameter functions for cardiac motion analysis from tagged mri data. 1996.

11. Alistair A. Young. Model tags: Direct 3D tracking of heart wall motion from tagged MR images. Lecture Notes in Computer Science, 1496:92-101, 1998. 